\title{
Enhancing Participation of Older Women in Surgical Trials
}

\author{
Patricia S Goode, MD, MSN, Mary P FitzGerald, MD, FACS, Holly E Richter, MD, PhD, \\ William E Whitehead, PhD, Ingrid Nygaard, MD, MS, Patricia A Wren, PhD, MPH, Halina M \\ Zyczynski, MD, Geoffrey Cundiff, MD, FACS, Shawn Menefee, MD, Judith M Senka, RNC, \\ Xin Gao, MS, Anne M Weber, MD, MS, and for the Pelvic Floor Disorders Network \\ Department of Medicine (Goode) and Department of Obstetrics and Gynecology (Richter), \\ University of Alabama at Birmingham, Birmingham, AL; Birmingham/Atlanta Geriatric Research, \\ Education, and Clinical Center, Veterans Affairs Medical Center, Birmingham, AL (Goode); \\ Department of Obstetrics and Gynecology, Loyola University Medical Center, Maywood, IL \\ (FitzGerald); Department of Medicine, University of North Carolina at Chapel Hill, Chapel Hill, NC \\ (Whitehead); Department of Obstetrics and Gynecology, University of Utah Health Sciences \\ Center, Salt Lake City, UT (Nygaard); Department of Health Behavior/Health Education, Oakland \\ University, Rochester, MI (Wren); Department of Obstetrics and Gynecology, Magee-Women's \\ Hospital, University of Pittsburgh, Pittsburgh, PA (Zyczynski); Department of Obstetrics and \\ Gynaecology, University of British Columbia, Vancouver, Canada (Cundiff); Department of \\ Obstetrics and Gynecology, University of California, San Diego, CA (Menefee); Department of \\ Obstetrics and Gynecology, Evanston Hospital, Evanston, IL (Senka); Department of \\ Biostatistics, University of Michigan, Ann Arbor, MI (Gao); and the National Institute of Child \\ Health and Human Development, NIH, Bethesda, MD (Weber).
}

\section{Abstract \\ BACKGROUND—Older participants are often excluded from clinical trials, precluding a representative sample.}

STUDY DESIGN-Using qualitative and quantitative methods, we examined recruitment and retention of older women with pelvic organ prolapse in two surgical trials: the randomized Colpopexy And Urinary Reduction Efforts (CARE) study and the Longitudinal Pelvic Symptoms and Patient Satisfaction After Colpocleisis cohort study. Using focus groups, we developed a questionnaire addressing factors facilitating and impeding the recruitment and retention of older study participants and administered it to research staff. Enrollment-to-surgery ratios, missed visit rates, and dropout rates for older and younger participants were compared using Fisher's exact test, with cut-points of 70 and 80 years for the CARE and Colpocleisis studies, respectively.

RESULTS-Questionnaires were completed by 23 physician investigators and 11 nurses or coordinators (92\% response rate). Respondents indicated it was more difficult to recruit older research participants (32\%), obtain informed consent (56\%), and retain participants to study completion (50\%). Challenges to recruitment included caregiver involvement in the decision to

\footnotetext{
(C) 2008 by the American College of Surgeons

Correspondence address: Patricia S Goode, MD, University of Alabama at Birmingham, Birmingham VA Medical Center, 11G, $700 \mathrm{~S}$ $19^{\text {th }}$ St, Birmingham, AL, 35233.

Disclosure Information: Nothing to disclose.

Author Contributions

Study conception and design: Goode, FitzGerald, Richter, Whitehead, Nygaard, Weber

Acquisition of data: Goode, FitzGerald, Richter, Whitehead, Nygaard, Wren, Zyczynski, Cundiff, Menefee, Senka, Weber

Analysis and interpretation of data: Goode, FitzGerald, Richter, Whitehead, Nygaard, Wren, Zyczynski, Cundiff, Menefee, Senka,

Gao, Weber

Drafting of manuscript: Goode, FitzGerald, Richter, Whitehead, Nygaard, Wren, Zyczynski, Cundiff, Menefee, Weber

Critical revision: Goode, FitzGerald, Richter, Whitehead, Nygaard, Wren, Zyczynski, Cundiff, Menefee, Senka, Weber
} 
participate and participant comorbidities. Perceived barriers to retention were transportation, caregiver availability, and participant fatigue. Data quality was challenged by sensory and cognitive impairment, resulting in a change from telephone interviews to in-person visits in the Colpocleisis study. Older participants did not have higher dropout rates than younger participants. There were no differences in missed in-person visits or telephone interview rates between age groups.

CONCLUSIONS-Strategies, albeit unstudied, could assist investigators in planning surgical trials that successfully enroll and retain older women.

The most robust manner in which clinical researchers can demonstrate the effectiveness of an intervention is through the use of randomized clinical trials or well-planned cohort studies. The success of these efforts depends in large part on recruiting and retaining a representative target population in adequate numbers to answer the scientific question. One particular challenge is including representative numbers of older participants in trials of therapies that will be prescribed for older persons. Older patients are often specifically excluded from clinical trials. ${ }^{1,2}$ Bugeja and colleagues ${ }^{3}$ reported that one-third of original research articles in major medical journals excluded elderly patients without justification.

Even when older patients are targeted for inclusion, their recruitment into surgical trials is particularly challenging. There is a growing body of literature exploring the challenges facing researchers interested in recruiting and retaining older adults in clinical trials and intervention studies. ${ }^{1,4-9}$ The consensus is that it is more difficult to recruit older participants than younger participants and that older women are more difficult to recruit than older men. ${ }^{10}$ Retention of older participants in trials after enrollment is reported to be comparable to retention of younger participants, ${ }^{4,11-13}$ but there are challenges to retention that are unique to the elderly-especially their dependence on family and caregivers for transportation. Another concern for the participation of older participants is data quality and particularly the effects of impairments in memory, hearing, and vision on responses to questionnaires and interviews.

The pressing need for surgical outcomes data in older patients is apparent as one considers the shift in US demographics. By the year 2030, individuals aged 65 and older, the majority of whom are women, will constitute nearly $20 \%$ of the population, an increase from the current proportion of $12 \% .{ }^{14}$ Pelvic floor disorders, including urinary incontinence, fecal incontinence, and pelvic organ prolapse, increase in prevalence as the average age of the population increases. ${ }^{15}$ More than 200,000 operations are performed yearly to treat pelvic organ prolapse alone, and the majority of these procedures are done on older women. ${ }^{16}$ The estimated demand for care of pelvic floor disorders has been projected to increase by $45 \%$ over the next 30 years, paralleling the changing population demographic. ${ }^{15}$ Outcomes data that include representative numbers of older women undergoing operations are essential to guide practice.

The purpose of this article was to outline the experience of the NIH Pelvic Floor Disorders Network (PFDN) with recruitment and retention of older women into two large, multisite urogynecologic surgical clinical trials. The primary data for this article came from a network-wide survey of physician investigators, study coordinators, and research nurses. We aimed to identify obstacles to recruitment and retention of older women in clinical trials and strategies for overcoming these barriers. 


\section{METHODS}

The Institutional Review Boards at all clinical sites and the Data Coordinating Center approved both clinical trials and this study of recruitment and retention. Both clinical trials were approved by the PFDN Data and Safety Monitoring Board.

\section{The CARE study}

The Colpopexy And Urinary Reduction Efforts (CARE) study, conducted between 2002 and 2007, was a randomized clinical trial in women without symptomatic stress incontinence undergoing sacrocolpopexy for pelvic organ prolapse, with or without the addition of a Burch retropubic urethropexy. The full methods of the CARE trial and its primary outcomes have been previously published. ${ }^{17,18}$ Participants were drawn from women with symptomatic pelvic organ prolapse presenting to the clinical PFDN sites. Women were eligible if they planned sacrocolpopexy for stages II-IV pelvic organ prolapse (per pelvic organ prolapse quantitative system [POP-Q] $)^{19}$ and they answered "never" or "rarely" to six of the stress incontinence questions from the Medical, Epidemiologic, and Social Aspects of Aging (MESA) questionnaire. ${ }^{20}$ Randomization occurred at the time of operation, after the participant was anesthetized. We followed participants for 2 years after surgery.

\section{The Colpocleisis study}

The Longitudinal Pelvic Symptoms and Patient Satisfaction After Colpocleisis study, conducted between 2004 and 2007, was a cohort study of women undergoing obliterative surgery (vaginal closure) to treat stages III-IV pelvic organ prolapse. Participants were enlisted from women electing colpocleisis at the participating PFDN sites. Participants were required to be community dwelling and mentally competent. We used the Short Portable Mental Status Questionnaire ${ }^{21}$ to screen potential participants for whom cognitive function was uncertain and for all participants over age 75 . We followed participants for 1 year after surgery.

\section{Analyses}

Both quantitative and qualitative methods were used to estimate differences in recruiting and retaining older women in the two surgical clinical trials. Qualitative analysis was conducted in two stages: focus groups and questionnaires. We initially conducted two focus groups to provide a foundation on which to optimally develop a questionnaire to administer to study coordinators, research nurses, and physician investigators conducting these studies. The purpose of the focus groups was to capture major themes related to the involvement of older women in these two surgical trials. Both focus groups involved investigators, research nurses, and study coordinators, who were asked their ideas about barriers to recruitment and retention of older participants, with special emphasis on any adaptations they had to make to involve older women in the two studies. Major themes were then developed into a simple questionnaire that was distributed electronically to all CARE and Colpocleisis physician investigators, research nurses, and study coordinators.

Respondents were asked whether they thought it was more difficult to recruit older research participants to CARE or Colpocleisis than it was to recruit younger participants, and they were asked to elaborate on their answers. They were also asked to describe specific challenges in recruiting older women and to list any techniques they used for recruiting, obtaining consent, and retaining older participants that they did not usually use with younger participants. They were then prompted to indicate and comment on whether any of the common themes identified during the focus groups were a problem while working with older study participants. Respondents were also asked to add or suggest any other special issues they thought relevant. Responses to the questionnaire were then compiled, and a 
working group identified the themes related to working with older participants and successful solutions.

To further describe the experience of the study network on recruiting older versus younger women into these two surgical trials, a quantitative analysis comparing dropout rates between enrollment and operation, dropout rates after operation, missed visit rates, and missed telephone interview rates was conducted. For the CARE study, participants were divided into cohorts aged less than 70 years and aged 70 years or older. The Colpocleisis participants were older, so we were able to compare retention and missed visit rates among participants aged less than 80 years to rates among participants aged 80 or older. CARE did not have sufficient participants older than 80 years ( 3 of 343; $0.9 \%$ ) nor Colpocleisis younger than 70 years $(15$ of $169 ; 8.9 \%)$ to divide each study group into 3 age cohorts. We considered a uniform cut-point of 75 years for both studies, but only 28 of 343 , or $8.2 \%$, of participants in CARE were 75 and older. Fisher's exact test was used to compare dropout and missed visit rates between the two age groups in each study.

\section{RESULTS \\ Demographics}

There were 343 participants enrolled in the CARE study. Ages ranged from 31 to 83 years, with 276 participants younger than 70 years old, 64 participants 70 to 79 years old, and 3 participants 80 years and older. Race distribution was as follows: $90.1 \%$ Caucasian, $5.8 \%$ African American, $0.6 \%$ American Indian/Alaska native, $0.3 \%$ Asian, and 3.2\% other. Ethnic origin included 3.2\% Hispanic or Latino participants.

There were 169 participants enrolled in the Colpocleisis study. Ages ranged from 64 to 93 years, with 15 participants younger than 70 years old, 91 participants 70 to 79 years old, and 63 participants 80 years and older. Race distribution was as follows: $92.3 \%$ Caucasian, $6.5 \%$ African American, $0.6 \%$ American Indian/Alaska native, and $0.6 \%$ other. Ethnic origin included $1.8 \%$ Hispanic or Latino participants.

\section{Focus groups and survey}

Two focus groups were conducted, during which major themes of working with older participants were identified (Table 1). Some themes were more applicable during specific phases of study participation, including recruitment, getting informed consent, and retention; others were more overarching. The final questionnaire queried all CARE and Colpocleisis physician investigators, research nurses, and study coordinators about special issues that arose in each phase of study participation. This was followed by a list of the themes identified by the focus groups that they could check if significant at their site and elaborate on solutions they developed or adopted.

Survey respondents included 23 physician investigators, 8 study coordinators, and 3 research nurses. The response rate was $92 \%$ (34 of 37). Thirty-two percent (11 of 34) of responders indicated it was more difficult to recruit older research participants. Sixty-seven percent (22 of 33, with 1 missing) agreed there were special challenges in recruiting older research participants, 56\% (19 of 34) identified difficulties in the informed consent process, and 50\% (15 of 30, with 2 uncertain and 2 missing) reported issues concerning retaining older research participants to the completion of the trials. Table 1 shows the percent who endorsed each of the themes that were identified in the focus groups as special problems in older participants.

Survey respondents offered multiple recruitment and retention strategies, which are summarized in Table 2.Transportation issues were common among older participants who 
do not drive and often depend on a friend or family member to get to study visits. The importance of conducting scheduled visits on time, scheduling study visits in advance (perhaps for the entire study), accurately specifying the time required for each appointment, tolerating multiple appointment changes, and thanking both the participant and the driver for their contributions to women's health were emphasized.

Study personnel deemed involving the family or significant others particularly important for older women, especially during the informed consent process when they are faced with long informed consent documents. The extra time required by older participants was an overarching theme. Participants sometimes needed extra visits in person or on the telephone so a family member could be involved in the informed consent process. Physicians often needed to describe the study to the patient initially, and then visit with the patient, again after study staff explained the study, to go over various aspects again and address any concerns that arose. Personal involvement of the physician was important, particularly in allaying the women's fears that they might not be good candidates for a research study. Study staff reported that extra time listening to the participants, particularly getting to know them and their families, and asking about significant life events at subsequent visits was important in participant retention.

Sensory impairment, both visual and auditory, was also very frequently mentioned. To accommodate for this, suggestions included the use of large-font forms, good lighting, and a quiet environment for visits. Memory impairment was a concern, and study staff suggested formally screening out women with dementia, because this may not be evident during standard screening. In the Colpocleisis study, the study with the older participants, mental status screening was done with the Short Portable Mental Status Questionnaire. ${ }^{21}$ Formal mental status assessment was not done in CARE. Several survey respondents remarked that even mild memory impairment made administration of multiple-choice instruments difficult on the telephone, frustrating both participants and study staff when these participants repeatedly could not remember all of the choices. In clinic, at some sites, administration of multiple-choice questions was facilitated by large-font "flash cards" with the choices listed.

\section{Recruitment, retention, and missed visit rates}

In the CARE study, the overall dropout rate was $5.8 \%$, with breakdown by age group as shown in Table 3. CARE participants younger than 70 years of age had a higher dropout rate between enrollment and randomization and surgery than the women 70 years of age and older $(7.6 \%$ versus $0 \%, \mathrm{p}=0.019)$. Overall dropout rates after randomization and surgery were similar between age groups: $6.7 \%$ of women less than 70 years old versus $4.5 \%$ of older women $(\mathrm{p}=0.776)$.

In the Colpocleisis study, the overall dropout rate was $10.6 \%$, with breakdown by age group as shown in Table 3. Dropout between enrollment and surgery in Colpocleisis participants was similar between age groups $(9.4 \%$ versus $9.5 \%, \mathrm{p}=1.000)$. The overall dropout rates after operation were also similar between age groups: $10.4 \%$ of women less than 80 years old versus $14.0 \%$ of women 80 years and older $(\mathrm{p}=0.502)$.

There were no differences in missed visit rates between groups. In the CARE study, younger women missed 66 of 1,014 (7\%) possible visits; women 70 years and older missed 19 of 266 (7\%) possible visits. In addition to the in-person visits, CARE participants had telephone interviews from the central Quality of Life Interviewing Center at 3, 6, 12, and 24 months. The missing rates were similar for the telephone interviews and for clinic visits (5\% versus $7 \%$ ) and identical between age groups. In the Colpocleisis study, telephone interviews by the Quality of Life Interviewing Center were attempted initially, but soon after beginning baseline data collection, the telephone interviewers reported difficulty in conducting 
telephone interviews with the older participants, particularly because of impaired hearing and memory. As soon as IRB permission could be obtained, quality-of-life data collection was done during in-person visits by the study coordinators. The missed visit rate in the Colpocleisis study was $7 \%$ (14 of 189 possible visits) in women younger than 80 years of age and $6 \%$ ( 7 of 112 possible visits) in older women.

\section{DISCUSSION}

\section{Recruitment}

Two-thirds of the investigators, coordinators, and research nurses in our network-wide survey reported special problems associated with recruiting older participants. But only onethird agreed that they found it more difficult to recruit older women. Other common themes identified by the focus group were also endorsed by only about half of the respondents. This most likely is because the focus groups were comprised of $95 \%$ nurse coordinators, and the survey respondents were $68 \%$ physician investigators. Usually physician investigators briefly present the study to their patients who are potential participants, and the bulk of the effort to enroll and retain the participants falls to the nurse coordinator. So only the study coordinators might be able to fully appreciate the recruitment and retention challenges.

The most frequent challenges to recruitment identified in our survey were hearing and visual impairment, transportation issues, the need to have family members attend visits and participate in the decision to enroll in the study, and other health problems that might make older women reluctant to enroll or cause difficulty with participation over an extended period of time.Other challenges were the woman's fear that she was not a "good candidate" for the research and difficulty in coping with long informed consent documents.

The specific strategies used at different study sites to overcome these challenges are summarized in Table 2. To address hearing and visual impediments, survey respondents recommended purchasing an inexpensive personal electronic amplifier with earphones, very similar in appearance to a portable digital music player, and using large-print documents. Our study coordinators recommend inviting family members to accompany participants, especially for appointments in which informed consent will be discussed, and if they are not available for the visit, to get them on the telephone with the participant present in the room. To deal with the participant's fear that she may not be an appropriate candidate, our teams recommend frequent, explicit reassurance by the investigator and establishment of good rapport.

The barriers to recruitment identified in our survey are consistent with what has been reported in other studies. ${ }^{10,22,23}$ But by recruiting patients from our own practices we were able to avoid a barrier that has been encountered when recruiting for treatment studies by advertisement or mailing to randomly selected people, namely, a recommendation against participation by the woman's personal physician. ${ }^{4,24}$

\section{Retention}

Several studies have reported that, once enrolled, older participants are no more likely to drop out than younger participants. ${ }^{4,11-13}$ In our study, although there was a higher dropout rate before operation in younger participants, the finding of one statistically significant difference was probably by chance. The finding of no difference at every other time point provides much stronger evidence for the conclusion that there was no difference in the retention rates or rates of missed visits between older and younger participants. But our survey revealed that half of the investigators and coordinators believed it was more difficult to retain older participants. This likely reflected the substantial increase in time required both to recruit and retain older participants compared with younger ones. Major barriers to 
retention of older women were transportation issues, fatigue during long study visits, hearing problems, and comorbid health problems that might interfere with participation. Strategies for dealing with these challenges are summarized in Table 2. Strategies for dealing with transportation problems include negotiating appointment times well in advance and sticking to schedule. Techniques for dealing with fatigue and communication problems include allowing sufficient time for responding, providing rest breaks, and being willing to space data collection out over multiple visits. The general strategy of establishing good rapport with participants by showing interest in other aspects of their lives has been mentioned by other investigators ${ }^{25}$ and was reinforced by our survey data.

\section{Data quality}

The question of whether self-reported data from older participants are as accurate as similar data collected from younger participants has rarely been addressed in published studies. Some of the barriers to participation identified in our survey, especially memory impairment but also impaired hearing and vision, are more closely related to data quality than to recruitment or retention. For mild forms of memory impairment that may make it difficult to respond to multiple-choice questions, we found it helpful to provide participants with flash cards listing possible responses during in-person interviews. Interviews conducted in this manner seemed to yield more reliable data than telephone interviews did because of hearing difficulty in addition to the issue of remembering multiple-choice responses. An alternative would be large-font questionnaires for the participant to follow during telephone interviews or to self-complete and return by mail.

Because of its significance for data quality, our investigative team recommends formal mental status testing to screen older participants. But despite using the Short Portable Mental Status Questionnaire, some of our participants in the Colpocleisis study were unable to remember the multiple-choice responses sufficiently to permit telephone administration of the quality-of-life instruments. This was not a problem with the participants of the CARE study, who were a decade younger. One respondent suggested the Mini-Cog, a brief standardized tool combining a three-item recall with a clock drawing test that they had used in another trial with older participants. ${ }^{26}$ Future research to establish the optimal tools to use to screen for cognitive impairment and the appropriate cut-points for participants to be able to provide accurate research data is warranted. More information on which cognitive level is actually incompatible with clinical trial participation and which lesser levels of impairment just need special accommodations in data collection methodology would be extremely useful. We believe that cognitive screening is a much better option than simply excluding research participants over an arbitrary age, as has been done in many studies. Including representative numbers of older participants in clinical trials is crucial so that the findings will be applicable to the population who will be receiving the treatments in the future.

\section{Limitations}

The survey report includes recommendations of experienced research team members who have successfully recruited and retained older women in surgical trials. It is possible that the lack of difference in recruitment and retention rates between older and younger participants we reported is a result of the specific techniques used for recruiting, consenting, and retaining older participants. But each specific recommendation requires validation to determine its relative benefit.

\section{Implications}

It is essential to include participants in clinical trials who are representative of the target populations likely to undergo the treatments under investigation. Recruiting a representative sample that includes adequate numbers of older women is difficult and costly: recruitment 
costs are estimated at $\$ 100^{22}$ to $\$ 300^{9}$ per participant and require an estimated 31 hours of staff time per recruited patient. ${ }^{4}$ Too often, recruitment strategies are poorly planned, underfunded, and have to be changed as the trial progresses. ${ }^{9}$ This may threaten the generalizability of the trial. For these reasons, investigators are encouraged to devote as much effort to planning their recruitment strategy as they do to designing the intervention and to dedicate adequate financial and human resources to recruitment and retention. This study provides guidance for the design of such recruitment and retention plans for surgical trials involving older women. Future research focusing on the relative effectiveness of specific recruitment strategies and their costs is warranted.

\section{Acknowledgments}

Supported by grants from the National Institute of Child Health and Human Development (U01 HD41249, U10 HD41268, U10 HD41248, U10 HD41250, U10 HD41261, U10 HD41263, U10 HD41269, and U10 HD41267).

\section{REFERENCES}

1. Polito A, Meunier N, Andriollo-Sanchez M, et al. Screening and recruitment procedure of latemiddle aged and older subjects: the ZENITH study. Euro J Clin Nutr. 2005; 59:S8-S12.

2. Morse AN, Labin LC, Young SB, et al. Exclusion of elderly women from published randomized trials of stress incontinence surgery. Obstet Gynecol. 2004; 104:498-503. [PubMed: 15339759]

3. Bugeja G, Kumar A, Banerjee AK. Exclusion of elderly people from clinical research: a descriptive study of published reports. BMJ. 1997; 315:1059-1061. [PubMed: 9366735]

4. Vogt TM, Ireland CC, Black D, et al. Recruitment of elderly volunteers for a multicenter clinical trial: the SHEP pilot study. Contr Clin Trials. 1986; 7:118-133.

5. Gill TM, McGloin JM, Gabbauer EA, et al. Two recruitment strategies for a clinical trial of physically frail community-living older persons. J Am Geriatr Soc. 2001; 49:1039-1045. [PubMed: 11555064]

6. Silagy CA, Campion K, McNeil JJ, et al. Comparison of recruitment strategies for a large-scale clinical trial in the elderly. J Clin Epidemiol. 1991; 44:1105-1114. [PubMed: 1941003]

7. Gallo JJ, Bogner HR, Straton JB, et al. Patient characteristics associated with participation in a practice-based study of depression in late life: the Spectrum study. Intl J Psych Med. 2005; 35:4157.

8. Wittink MN, Oslin D, Knott KA, et al. Personal characteristics and depression-related attitudes of older adults and participation in stages of implementation of a multi-site effectiveness trial (PRISME). Intl J Geriatr Psych. 2005; 20:927-937.

9. Ory MG, Lipman PD, Karlen PL, et al. Recruitment of older participants in frailty/injury prevention studies. Prev Sci. 2002; 3:1-22. [PubMed: 12002555]

10. Boult C, Pirie P, Boult L, Morishita L. Soliciting defined populations to recruit samples of highrisk older adults. J Gerontol A Biol Sci Med Sci. 1998; 53:M379-M384. [PubMed: 9754144]

11. Petrovich H, Byington R, Bailey G, et al. Systolic Hypertension in the Elderly Program (SHEP): baseline characteristics of the randomized sample: Part 2: screening and recruitment. Hypertension. 1991; 17:16-23.

12. Carter WB, Elsward K, Malmgren J, et al. Participation of older adults in health programs and research: a critical review. Gerontologist. 1991; 31:584-592. [PubMed: 1778481]

13. Carew BD, Ahn SA, Boichot HD, et al. Recruitment strategies in the studies of left ventricular dysfunction (SOLVD): strategies for screening and enrollment in two concurrent but separate trials. Controlled Clin Trials. 1992; 13:325-338. [PubMed: 1330437]

14. The state of aging and health in America. [Accessed April 4, 2007] Centers for Disease Control and Prevention. 2007. Available at: www.cdc.gov

15. Luber KM, Boreo S, Choe JY. The demographics of pelvic floor disorders; current observations and future projections. Am J Obstet Gynecol. 2001; 184:1496-1501. [PubMed: 11408873]

16. Boyles SH, Weber AM, Meyn L. Procedures for pelvic organ prolapse in the United States, 19791997. Am J ObstetGynecol. 2003; 188:108-115. 
17. Brubaker L, Cundiff GW, Fine $P$, et al. A randomized trial of colpopexy and urinary reduction efforts (CARE): design and methods. Control Clin Trials. 2003; 24:629-642. [PubMed: 14500059]

18. Brubaker L, Cundiff GW, Fine P, et al. Abdominal sacrocolpopexy with Burch colposuspension to reduce urinary stress incontinence. N Engl J Med. 2006; 354:1557-1566. [PubMed: 16611949]

19. Bump RC, Mattiasson A, Bo K, et al. The standardization of terminology of female pelvic organ prolapse and pelvic floor dysfunction. Am J Obstet Gynecol. 1996; 175:10-17. [PubMed: 8694033]

20. Diokno AC, Brock BM, Brown MB, Herzog AR. Prevalence of urinary incontinence and other urological symptoms in the non-institutionalized elderly. J Urol. 1986; 136:1022-1025. [PubMed: 3490584]

21. Roccaforte WH, Burke WJ, Bayer BL, Wengel SP. Reliability and validity of the Short Portable Mental Status Questionnaire administered by telephone. J Geriatr Psychiatry Neurol. 1994; 7:3338. [PubMed: 8192828]

22. Adams J, Silverman M, Musa D, Peele P. Recruiting older adults for clinical trials. Control Clin Trials. 1997; 18:14-26. [PubMed: 9055049]

23. DiBartolo MC, McCrone S. Recruitment of rural community-dwelling older adults: barriers, challenges and strategies. Aging Ment Health. 2003; 7:75-82. [PubMed: 12745386]

24. Jeffe DB, Binder EF, Williams DB, Kohrt WM. Frail older women's participation in a trial of hormone replacement therapy: perceived benefits and concerns. Menopause. 2001; 8:127-134. [PubMed: 11256873]

25. Tolmie EP, Mungall MMB, Louden G, et al. Understanding why older people participate in clinical trials: the experience of the Scottish PROSPER participants. Age Ageing. 2004; 33:374378. [PubMed: 15151909]

26. Borson S, Scanlan J, Brush M, et al. The Mini-Cog: a cognitive 'vital signs' measure for dementia screening in multi-lingual elderly. Int J Geriatr Psychiatry. 2000; 15:1021-1027. [PubMed: 11113982] 
Table 1

Barriers to Participation of Older Women in Surgical Trials

\begin{tabular}{llr}
\hline Category & Barrier & $\begin{array}{r}\text { Respondents } \\
\text { endorsing, \% }\end{array}$ \\
\hline Barriers to recruitment & Caregiver or family involvement in decision to participate & 53 \\
& Comorbid medical conditions & 41 \\
\hline & Comorbid medical conditions & 41 \\
\hline Barriers to retention & Transportation issues & 65 \\
& Caregiver or family attendance at visits with the study participant & 35 \\
& Fatigue during longer study visits & 47 \\
\hline \multirow{2}{*}{ Challenges to data quality } & Hearing impairment & 44 \\
& Visual impairment & 9 \\
& Memory impairment/dementia & 26 \\
\hline
\end{tabular}


Table 2

\section{Strategies for Recruitment and Retention of Older Female Participants in Surgical Trials}

\begin{tabular}{|c|c|c|}
\hline Themes & Strategies & \\
\hline \multirow[t]{9}{*}{ Reluctance to enroll in a study } & 1 & Multistep visit: \\
\hline & & $\begin{array}{l}\text { A. Physician briefly introduces the study and tells the potential participant } \\
\text { that she is a great candidate for the study. }\end{array}$ \\
\hline & & Research nurse thoroughly presents details of study involvement. \\
\hline & & $\begin{array}{l}\text { C. Physician returns to answer questions and asks the patient about her } \\
\text { understanding of study participation. }\end{array}$ \\
\hline & 2 & $\begin{array}{l}\text { Spend time, have patience, and develop a personal relationship with each older } \\
\text { participant. Take an active interest in her life. Listen to what she has to say and } \\
\text { remember it. Bring up something that she told you (grandchildren, vacations, and so } \\
\text { on) during the next visit. This lets her know that you remember her. }\end{array}$ \\
\hline & 3 & Smile. Reassure her that you will take good care of her. \\
\hline & 4 & A personal explanation is much more effective than a brochure or pamphlet. \\
\hline & 5 & $\begin{array}{l}\text { Emphasize the importance of the woman's contribution; particularly emphasize the } \\
\text { value of her insight and experience for future participants. }\end{array}$ \\
\hline & 6 & Always thank patients for their time and walk them out of the clinic. \\
\hline
\end{tabular}

Visits take longer than with younger participants

1 Time to explain research procedures versus usual care and to fully understand what is involved in study participation.

2 Time to reassure participants about their health and ability to participate.

3 Older participants love to talk.
1 Spend the extra time. Time spent with the older participant during recruitment usually determines retention and compliance.

2 Plan for longer visits.

3 Patience, patience, patience; older participants cannot be rushed.
1 Stay on schedule with visits. See participants promptly to prevent waiting.

2 If a participant becomes fatigued, allow a short break for tea, coffee, water, or a bathroom visit.

3 If a participant is tired, ask her if she would like to finish another day.

1 Identify the family member who usually participates in healthcare decisions.

2 Include the family member in enrollment discussions with the participant's permission-in person is preferable, even if it requires a repeat visit. Alternately, a speakerphone can be used with the patient in the room.

3 If the patient prefers, allow a family member to review the consent form before the patient signs it.

4 Encourage the participant or family member to call if any questions about the research study arise.

5 Clinic room needs seating for both family and patient.

6 Spend some time to talk with the family and get to know them.

Changing mind after initially agreeing to participate (usually after going home and talking to family or friends)
1 After it is apparent that the patient is inclined to participate, include a family member or other decision maker in the enrollment discussion.

2 Ask the participant if she is sure she wants to participate. Assure her it is permissible to decline.

Multiple health problems 


\begin{tabular}{|c|c|c|c|}
\hline Themes & & Strategies & \\
\hline 1 & $\begin{array}{l}\text { Older woman's concern of good } \\
\text { health status continuing until }\end{array}$ & 1 & $\begin{array}{l}\text { Physician reassures her that she is a good candidate for the study when inviting her } \\
\text { to participate. }\end{array}$ \\
\hline \multirow[t]{2}{*}{2} & $\begin{array}{l}\text { completion of the study. } \\
\text { Developing health problems }\end{array}$ & 2 & $\begin{array}{l}\text { Despite higher prevalence of health problems, the dropout rate is the same between } \\
\text { older and younger participants, so don't exclude older women. }\end{array}$ \\
\hline & $\begin{array}{l}\text { during the study or becoming a } \\
\text { caregiver for someone with } \\
\text { health problems and being } \\
\text { overwhelmed. }\end{array}$ & 3 & $\begin{array}{l}\text { Studies of incontinence should include provision to treat urinary tract infections and } \\
\text { possibly allow longer potential windows between visits. }\end{array}$ \\
\hline 3 & $\begin{array}{l}\text { More frequent urinary tract } \\
\text { infections interrupt the } \\
\text { evaluation and treatment. }\end{array}$ & & \\
\hline
\end{tabular}

\begin{tabular}{cl}
\hline Memory impairment \\
\hline $\mathbf{1} \quad \begin{array}{l}\text { Avoiding enrolling participants } \\
\text { with dementia who are unable } \\
\text { to adhere to the study protocol } \\
\text { or reliably provide outcomes } \\
\text { measures. }\end{array}$ \\
$\mathbf{2} \quad \begin{array}{l}\text { Difficulty of administering a } \\
\text { multiple-choice questionnaire } \\
\text { over the telephone; some older } \\
\text { participants can't remember the } \\
\text { choices. }\end{array}$
\end{tabular}

1 Screen for dementia with a short standardized tool such as the Mini-Cog, a threeitem recall with a clock drawing test. ${ }^{26}$ Include inability to pass as exclusion criterion.

2 If multiple-choice questions are used in assessment instruments, use mailed questionnaires with large print instead of telephone administration.

3 For in-person interviews, use flash cards with large font for multiple-choice questions.

\begin{tabular}{llll}
\hline Hearing impairment & $\mathbf{1}$ & $\begin{array}{l}\text { Purchase an inexpensive amplification device with a lightweight headset for the } \\
\text { clinic. }\end{array}$ \\
$\mathbf{2}$ & Ask participant to wear her hearing aid, if she has one, to the next visit. \\
$\mathbf{3}$ & Face the patient so she can use lip-reading cues. \\
$\mathbf{4}$ & Good lighting helps hearing-impaired persons lip read. \\
$\mathbf{5}$ & Speak slightly more slowly. \\
$\mathbf{6}$ & Provide a quiet environment. \\
7 & Provide a private room to accommodate louder conversations.
\end{tabular}

Visual impairment

1 Use large-font forms, including the consent form.

2 Provide good lighting.

1 Need for someone to drive participants to visits and fear of imposing, especially if driver needs to take off from work.

2 Fear of driving into the city.

3 Extended winter trips to escape the cold for participants in colder climates.

4 Transportation within the clinic may be a problem because of frailty.
1 Clarify time involvement.

2 Provide compensation for travel expenses.

3 Schedule study visits based on transportation availability, eg, same day as another appointment, such as a mammogram or with another physician.

4 Schedule visits far in advance, perhaps for the entire study.

5 Be prepared for multiple appointment changes.

6 If necessary, schedule visits outside of usual clinic times.

7 Don't change participant appointments once made unless they request it (eg, canceling clinics).

8 Stay on schedule so that the person driving the participant is not inconvenienced.

9 Thank the person driving the participant for his role in helping to advance women's health care.

10 Consider using nonurban satellite clinic locations.

11 If participants winter elsewhere, time enrollment to allow completion of study or for the travel window to coincide with a window of the protocol. 


\begin{tabular}{rlll}
\hline Themes & Strategies \\
\hline Longterm followup & $\mathbf{1 2}$ & $\begin{array}{l}\text { Escort participants to the laboratory, other places in the clinic that they need to go, } \\
\text { or to their car. }\end{array}$ \\
\hline $\mathbf{1} \quad \begin{array}{l}\text { When recovered from surgery } \\
\text { and feeling better, they may not } \\
\text { understand why more visits are } \\
\text { necessary. }\end{array}$ & $\mathbf{1}$ & $\begin{array}{l}\text { Remind about next study visit and purpose of the visit and the study each time they } \\
\text { are seen. }\end{array}$ \\
\hline $\begin{array}{l}\text { They may forget they are in a } \\
\text { study. }\end{array}$ & $\mathbf{2}$ & $\begin{array}{l}\text { Screen for occult dementia using a short screening test and exclude those failing the } \\
\text { screening. }\end{array}$ \\
\hline
\end{tabular}


